\title{
Consequences of Stopping and Restarting Leptin in an Adolescent with Lipodystrophy
}

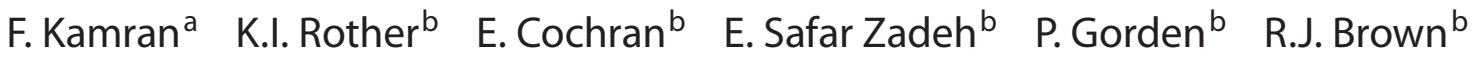 \\ ${ }^{a}$ National Institute of Child Health and Human Development, and ${ }^{\mathrm{b}}$ National Institute of Diabetes and Digestive \\ and Kidney Diseases, National Institutes of Health, Bethesda, Md., USA
}

\section{Established Facts}

- Acquired generalized lipodystrophy is a rare disorder characterized by leptin deficiency.

- Leptin replacement therapy effectively ameliorates the metabolic derangements of lipodystrophy, but does not cause regeneration of adipose tissue.

\section{Novel Insights}

- This case report highlights the significance of uninterrupted leptin therapy compared with intermittent treatment of patients with acquired generalized lipodystrophy.

- Lack of cosmetic changes such as restoration of subcutaneous fat may contribute to poor compliance or discontinuation of treatment by the patient.

- Discontinuation of leptin may unmask worsening metabolic abnormalities characteristic of the natural history of lipodystrophy.

- In addition to improvement of metabolic disease, restarting leptin therapy may enable normal pubertal progression.

\section{Key Words}

Lipodystrophy $\cdot$ Leptin $\cdot$ Nonalcoholic steatohepatitis ·

Diabetes $\cdot$ Adipose tissue $\cdot$ Adolescent

\section{Abstract \\ Background/Aims: Lipodystrophy encompasses a group of rare disorders characterized by deficiency of adipose tissue resulting in hypoleptinemia, and metabolic abnormalities including insulin resistance, diabetes, dyslipidemia, and}

nonalcoholic steatohepatitis. Leptin replacement effectively ameliorates these metabolic derangements. We report effects of leptin discontinuation and resumption in a child with acquired generalized lipodystrophy. Methods: Intermittent treatment with leptin with follow-up over 5 years. Results: Pretreatment metabolic abnormalities included insulin resistance, hypertriglyceridemia and steatohepatitis. Leptin was started at the age of 10 years. After 2 years, the family requested discontinuation of leptin due to lack of visible physical changes. Nine months later, worsened metabolic

\section{KARGER}

Fax +41 613061234

E-Mail karger@karger.ch

www.karger.com
(C) 2012 S. Karger AG, Basel

$1663-2818 / 12 / 0786-0320 \$ 38.00 / 0$

Accessible online at:

www.karger.com/hrp
Rebecca J. Brown

Building 10/CRC, Rm 6-5942

10 Center Drive, Bethesda, MD 20892 (USA)

Tel. +1 3015940609

E-Mail brownrebecca@mail.nih.gov 
abnormalities and arrest of pubertal development were observed. Leptin was restarted, followed by improvements in metabolic parameters. Laboratory changes (before vs. 6 months after restarting leptin) were: fasting glucose from 232 to $85 \mathrm{mg} / \mathrm{dl}$, insulin from 232 to $38.9 \mu \mathrm{U} / \mathrm{ml} \mathrm{HbA}_{1 \mathrm{c}}$ from 7.5 to $4.8 \%$, triglycerides from 622 to $96 \mathrm{mg} / \mathrm{dl}$, ALT from 229 to $61 \mathrm{U} / \mathrm{l}, \mathrm{AST}$ from 91 to $18 \mathrm{U} / \mathrm{l}$, and urine protein:creatinine ratio from 5.4 to 0.3 . Progression of puberty was observed 1 year after restarting leptin. Conclusion: Initial leptin therapy likely prevented progression of metabolic abnormalities. Treatment discontinuation led to rapid metabolic decomposition and pubertal arrest. Reintroduction of leptin reversed metabolic abnormalities and allowed normal pubertal progression.

Copyright $\odot 2012$ S. Karger AG, Basel

\section{Introduction}

Congenital and acquired lipodystrophies are characterized by partial or generalized loss of adipose tissue. Patients with acquired generalized lipodystrophy (AGL) have normal body fat at birth, and experience a gradual disappearance of fat during childhood and adolescence. Usually the onset of lipodystrophy occurs over months to years, and the resultant loss of fat produces a muscular appearance. Females are affected approximately three times more often than males.

The metabolic abnormalities of AGL exhibit considerable individual variability, and include impaired glucose tolerance or diabetes mellitus, severe fasting and/or postprandial hyperinsulinemia, reduced serum concentrations of the adipocyte-derived hormones leptin and adiponectin, as well as hypertriglyceridemia and low levels of serum HDL cholesterol. In addition to the loss of subcutaneous fat, clinical signs of lipodystrophy include acanthosis nigricans and hepatosplenomegaly. The liver enlargement can be mild to massive. Liver disease ranges from mild hepatic steatosis (nonalcoholic fatty liver disease (NAFLD)) with or without transaminase elevation, to nonalcoholic steatohepatitis (NASH), to cirrhosis with portal hypertension and esophageal varices $[1$, 2].

Due to the partial or complete absence of adipose tissue (and hence, the adipokine leptin), lipodystrophies represent a model for understanding the actions of leptin in a state of deficiency and replacement. Leptin replacement in lipodystrophy reduces food intake $[3,4]$ and ameliorates most metabolic abnormalities, including insulin resistance $[4,5]$, diabetes [5-7], hypertriglyceridemia [6,
7] and hepatic steatosis $[2,4]$. We report the long-term effects of discontinuation and effective reinstitution of leptin treatment in a child with AGL.

\section{Case Report}

An 8-year-old Caucasian girl was enrolled in the ongoing clinical protocol (Clinical Trial Identifier NCT00025883) for treatment of lipodystrophy with leptin at the National Institutes of Health (NIH) after appropriate assent and consent had been obtained. She had an unremarkable prenatal and neonatal course. Developmental history was within normal limits. At 3 years of age, she developed dimpling in her lower extremities localized to the inner thighs. This gradually spread to include both upper and lower extremities, and was associated with a loss of subcutaneous fat. In the following years, she also lost subcutaneous fat in the trunk region with sparing of her face and neck. She developed a voracious appetite and acanthosis nigricans in the axillae, antecubital fossae and neck. At the time of her initial NIH evaluation at age 8 , she had significant insulin resistance, hypoleptinemia, hypertension, hypertriglyceridemia and elevated transaminases (table 1) coupled with decreased liver echogenicity on ultrasound, consistent with fatty liver disease. Her only medication was fish oil and she followed a low-fat diet. Physical examination revealed loss of subcutaneous fat in the trunk and extremities and muscular appearance with preservation of subcutaneous fat in the face and neck. A diagnosis of AGL was reached based on the progressive loss of body fat that started after age 3 , lack of genetic mutation, low leptin level and typical metabolic abnormalities associated with lipodystrophy.

The patient returned for follow-up 2 years later (age 10), at which time she was Tanner stage II for breasts and Tanner I for pubic hair, with otherwise little change in her physical examination and metabolic parameters (table 1). In the interim she had started taking an ACE inhibitor for hypertension. Her bone age was 20 months advanced compared to her chronological age (fig. 1, growth chart). There was no evidence for an underlying autoimmune condition at either the initial or follow-up visit. A liver biopsy showed diffuse, predominantly microvesicular steatosis with mild inflammatory activity and bridging fibrosis, consistent with NASH (NASH score of 5 out of 8 , with 8 being the highest severity [8]). In light of the presence of NASH, hypertriglyceridemia, and insulin resistance, she was found to be an appropriate candidate for leptin therapy. Treatment was initiated with recombinant metreleptin (r-metHuLeptin), at a dose of 0.06 $\mathrm{mg} / \mathrm{kg} /$ day administered subcutaneously as previously described [6]. The patient tolerated leptin therapy well with stable metabolic status (table 1) with approximately $70 \%$ compliance based on requested medication refills.

Physical examination at age 12, after 2 years of leptin therapy, revealed generalized loss of fat, including loss of mechanical fat from the palms and soles, mild acanthosis, mild hepatomegaly, height $>97$ th percentile, weight $75-90$ th percentile (fig. 1), elevated systolic blood pressures of 129-152 $\mathrm{mm} \mathrm{Hg}$ (95th percentile for age, gender, and height equals $126 \mathrm{~mm} \mathrm{Hg}$ ), and Tanner stage III pubertal development. Her medications at that time included leptin, metformin and an ACE inhibitor. Liver ultrasound showed unchanged fatty infiltration, and a liver biopsy revealed moderate 
Table 1. Clinical and biochemical parameters

\begin{tabular}{|c|c|c|c|c|c|c|c|c|}
\hline $\begin{array}{l}\text { Clinical parameter } \\
\text { (normal range) }\end{array}$ & \multicolumn{8}{|l|}{ Age } \\
\hline Fasting insulin, $\mu \mathrm{U} / \mathrm{ml}(6-27)$ & 112 & 57.6 & 193 & 42.4 & 111 & 235 & 38.9 & 51.8 \\
\hline $\mathrm{HbA}_{1 \mathrm{c}}, \%(4.8-6.4)$ & 4.8 & 4.6 & 4.5 & 4.8 & 5.0 & 7.5 & 4.8 & 5.2 \\
\hline Triglycerides, mg/dl & 292 & 108 & & 122 & 137 & 622 & 96 & 57 \\
\hline ALT, U/l (6-41) & 91 & 90 & 68 & 170 & 282 & 229 & 61 & 129 \\
\hline AST, U/1 (9-34) & 48 & 48 & 36 & 64 & 101 & 91 & 18 & 44 \\
\hline IGF-1, ng/ml (see note) & 107 & 186 & & 215 & 218 & 98 & 347 & 221 \\
\hline Urine protein (negative) & negative & negative & & negative & negative & $3+$ & $1+$ & \\
\hline
\end{tabular}

$\mathrm{HbA}_{1 \mathrm{c}}=$ Hemoglobin $\mathrm{A}_{1 \mathrm{c}} ;$ ALT = alanine aminotransferase; AST = aspartate aminotransferase; IGF-1 = insulin-like growth factor-1. IGF-1 normal range according to Tanner stages in females: Tanner I: 49-342, Tanner II: 115-428, Tanner III: 145-760, Tanner IV: $244-787$.

steatosis with moderate inflammation and bridging fibrosis. The NASH pathologic score was unchanged. Due to the perceived lack of improvement, especially the lack of change in her physical appearance, the patient and her family requested discontinuation of leptin. After a detailed discussion among the physicians and family, therapy was discontinued and a follow-up visit was scheduled for close monitoring of the metabolic profile.

Following leptin discontinuation the patient again developed a voracious appetite and increased acanthosis. A follow-up visit was planned for 6 months, however the patient was unable to return until 9 months later. At her NIH clinic visit 9 months after leptin discontinuation, clinical and biochemical parameters had markedly deteriorated. She had severe acanthosis nigricans on the neck, axillae, antecubital fossae and inguinal region, as well as significant hepatomegaly, with a liver span $9.5 \mathrm{~cm}$ below the costal margin. Her pubertal development had not progressed over the course of 9 months. Laboratory results confirmed overt diabetes (fasting glucose $232 \mathrm{mg} / \mathrm{dl}, \mathrm{HbA}_{\mathrm{lc}} 7.5 \%$ ) with hyperinsulinemia (serum insulin $235 \mu \mathrm{U} / \mathrm{ml}$ ), hyperlipidemia (triglyceride $622 \mathrm{mg} / \mathrm{dl}$ ), ALT $229 \mathrm{U} / \mathrm{l}$, AST $91 \mathrm{U} / \mathrm{l}$, and an elevated urine protein:creatinine ratio of 5.4 (table 1 ).

Due to this clinical deterioration, the decision was made to restart leptin therapy. Follow-up visits 6 and 12 months after restarting leptin showed dramatic improvements in clinical and laboratory parameters (table 1). Her hypertension continued to worsen despite leptin, requiring the addition of a calcium channel blocker to the ACE inhibitor. An extensive workup for causes of hypertension (including renal, vascular, and endocrine) was negative.

\section{Discussion}

We have documented the long-term effects of introducing, pausing, and restarting leptin therapy in a child with acquired, generalized lipodystrophy. Our patient began leptin injections at the age of 10 years in order to treat complications of lipodystrophy, including hypertriglyceridemia, insulin resistance and NASH. This treatment was continued for 2 years during which time the patient's metabolic profile was stable. We speculate, however, that the patient and her family had expected a reversal of her adipose tissue loss, despite having been informed that this was not an expected effect of treatment. Upon the family's request, leptin treatment was discontinued and marked worsening of the patient's clinical status occurred, including the onset of overt diabetes. The patient's dramatic worsening during her time off of leptin may have been exaggerated by the physiologic insulin resistance of puberty, as she was mid-pubertal during this period. Upon reintroduction of leptin, clinical improvement was documented within 6 months.

Clinical management of AGL remains a challenge. Insulin resistance and other metabolic disturbances such as diabetes mellitus and hypertriglyceridemia appear early in the course of the disease [1] and, if untreated, progressively worsen and result in early death. Various therapeutic measures have been explored to improve the physical 


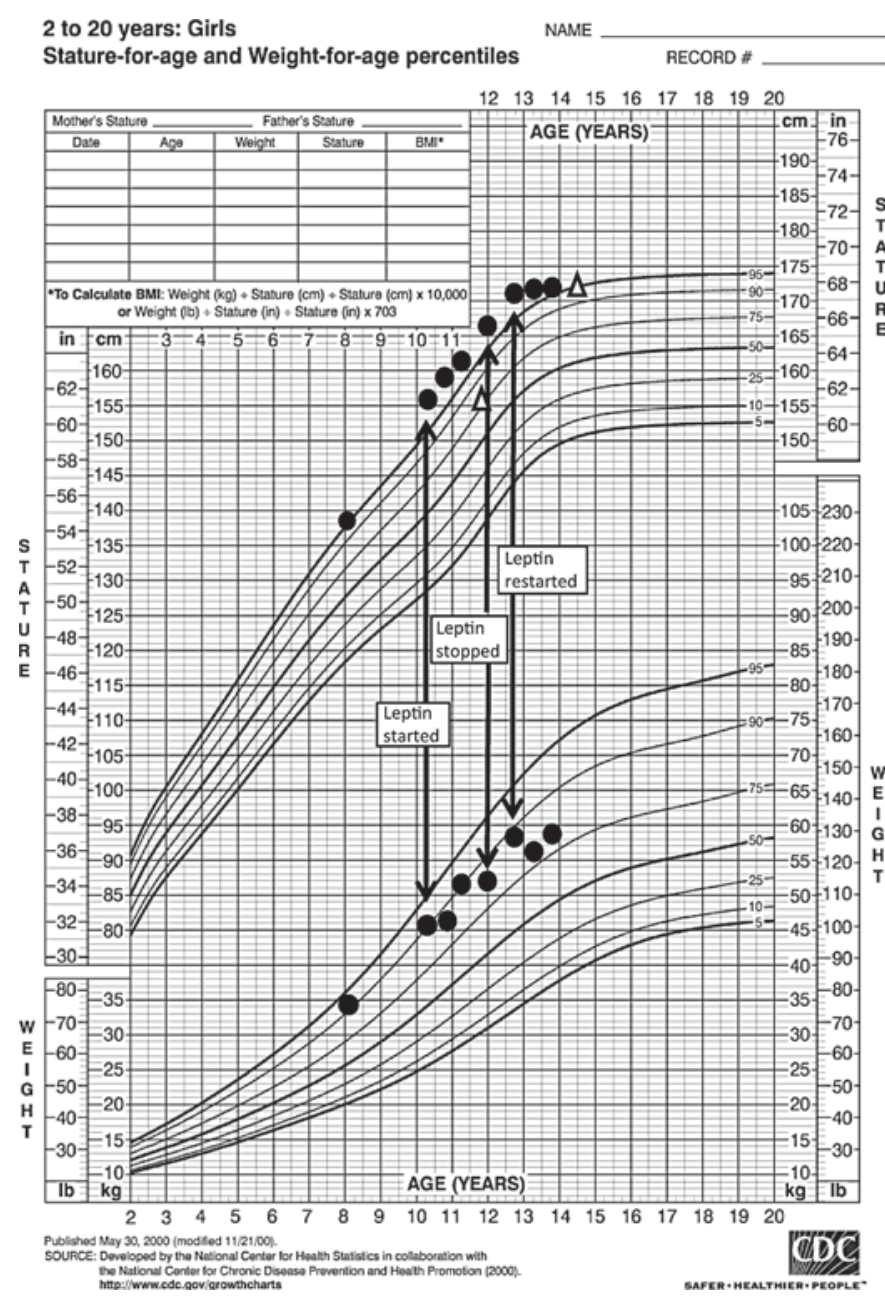

Fig. 1. Growth chart showing height and weight of the patient from age 8 to age 13 years. Height and weight are shown as filled black circles, and bone ages as open triangles. Treatment with leptin was initiated at age 10 years 4 months. Treatment was discontinued at age 12 years and then restarted at age 12 years 9 months.

and metabolic abnormalities associated with the condition. High doses of insulin, thiozolidinediones, metformin, and fibrates have all been used for metabolic improvements $[1,2,6,9]$. However, unlike other therapies, leptin replacement normalizes energy balance, which in turn significantly ameliorates numerous metabolic abnormalities $[3,5,7]$.

Leptin is a $16-\mathrm{kDa}$ protein of 167 amino acids. The $\mathrm{Ob}$ (Lep) gene is located on chromosome 7 in humans [10]. Leptin is secreted predominantly by adipose tissue [11], but is also expressed in placenta [12], and other tissues. Leptin has been shown to act at the hypothalamus in mice, inhibiting the synthesis of orexigenic peptides including agouti-related protein [13] and neuropeptide Y. Leptin has a circulating half-life of approximately $30 \mathrm{~min}$ and is released in a pulsatile manner from adipose tissue. There is a circadian rhythm in circulating levels with a nocturnal elevation in concentration $[14,15]$. Many adipokines have now been identified, however leptin is the only one to be administered in a human deficiency state.

The major physiologic effects of leptin in patients with lipodystrophy are reduction of hyperphagia [3, 7], lowering of serum triglycerides, improvement in fatty liver disease, decreased insulin resistance [6], and normalization of the hypothalamic-pituitary-ovarian axis [16], permitting menstrual cycling and fertility. Our patient's hyperphagia was clearly temporally correlated with her leptin use. Prior to the initiation of leptin, she was consuming 3,000-4,000 calories/day. During follow-up evaluations while receiving leptin her appetite was significantly decreased. The rise in serum insulin observed in this patient between the ages of 10 and 12 years while receiving leptin may have been the result of somewhat imperfect compliance, coupled with the physiologic insulin resistance of puberty. She also had elevated liver enzymes during this period, without an improvement in NASH scoring. It is possible that the fluctuations in her liver enzymes during leptin treatment may have been due to either imperfect compliance, or the coexistence of an additional liver disease, autoimmune hepatitis, which has been described in patients with AGL $[17,18]$.

In addition to its vital role in maintaining energy homeostasis, leptin is also considered to be a signal in the initiation of puberty, indicating a link between nutritional status and reproductive capacity. Leptin administration permits the progression of appropriately timed puberty, but does not appear to cause precocious activation of the pubertal process in younger children [19]. While leptin replacement appears to be necessary for normal pubertal development in patients with congenital leptin deficiency, the role of exogenous leptin in pubertal progression in patients with lipodystrophy is less clear. Leptin permits normal menstrual cycles in the majority of postpubertal females with lipodystrophy. In our patient, leptin administration and discontinuation were temporally correlated with pubertal progression. Our patient had arrest of pubertal development during the 9-month period in which she was not receiving leptin. However, because this patient had slow progression of $\mathrm{pu}$ berty even while on leptin (progressing from Tanner stage II to III over 2 years), the lack of pubertal progression over 9 months of leptin withdrawal may simply rep- 
resent this patient's natural, slow, progression. Follow-up evaluation 1 year after restarting leptin revealed significant pubertal advancement, with progression of both breast development and pubic hair from Tanner stage III to Tanner stage IV. She had her first menstrual period 2 weeks prior to the follow-up visit 12 months after resumption of leptin. Leptin appears to be required for normal menstrual cycles, but its role in progression of breast development remains to be elucidated. Of note, patients with lipodystrophy have tall stature despite advanced bone age as seen in our patient. As shown in the growth chart she continued to grow at or above the 97th percentile despite advanced bone age. As has been previously reported, leptin treatment was associated with weight loss or slowed weight gain [3].

The presence of hypertension has occasionally been reported in children with various types of lipodystrophy, including both congenital [20] and acquired [1] generalized lipodystrophy, but neither its precise prevalence nor the pathophysiologic mechanism have been elucidated. It is thought that it may be a component of the metabolic syndrome picture in these patients, analogous to hypertension in the common, obesity-associated metabolic syndrome. Interestingly, hypertension in the context of peroxisome proliferator-activated receptor- $\gamma$ (PPAR $\gamma$ ) mutation (a form of familial, partial lipodystrophy) has been looked at in more detail. Tsai et al. [21] showed that the PPAR $\gamma$ mutation independently affects other pathways, in particular the renin-angiotensin system. Both human PPAR $\gamma$ P467L heterozygotes and PPARy P465L heterozygous mice are hypertensive, despite the fact that the mice are minimally insulin-resistant. However, no specific etiology has been identified for hypertension observed in patients with acquired types of lipodystrophy.

Although leptin is effective at improving many of the metabolic disturbances associated with lipodystrophy, it cannot regenerate missing adipose tissue. Patients with lipodystrophy may experience psychological distress and physical discomfort due to the loss of subcutaneous fat, presence of acanthosis nigricans, xanthomas, hirsuitism and muscular appearance. In addition, loss of plantar fat can result in the development of painful callosities limiting physical mobility. Many patients, especially those with acquired lipodystrophy, may have false expectations that leptin will restore their subcutaneous adipose tissue and normalize their appearance. It is essential to counsel patients and their families that leptin effectively reduces the appearance of acanthosis secondary to insulin resistance, but it cannot restore the lost subcutaneous fat. Lack of improvement in subcutaneous adipose tissue may lead to the perception that leptin is not effective and may be a cause of nonadherence to the treatment regimen.

In summary, loss of subcutaneous fat with progressively worsening metabolic profile is the hallmark of lipodystrophy. Successful therapy with leptin has changed the natural history of this disease. Because lipodystrophy-associated metabolic derangements tend to worsen over time without treatment, leptin therapy may halt the progression of these manifestations, rather than reversing them, as was observed during the first period of leptin treatment in our patient. Discontinuation of leptin allows the natural history of the disease to manifest once again.

\section{References}

-1 Misra A, Garg A: Clinical features and metabolic derangements in acquired generalized lipodystrophy: case reports and review of the literature. Medicine (Baltimore) 2003;82: 129-146.

$>2$ Javor ED, Ghany MG, Cochran EK, Oral EA, DePaoli AM, Premkumar A, et al: Leptin reverses nonalcoholic steatohepatitis in patients with severe lipodystrophy. Hepatology 2005;41:753-760.

3 Moran SA, Patten N, Young JR, Cochran E, Sebring N, Reynolds J, et al: Changes in body composition in patients with severe lipodystrophy after leptin replacement therapy. Metabolism 2004;53:513-519.
4 Petersen KF, Oral EA, Dufour S, Befroy D, Ariyan C, Yu C, et al: Leptin reverses insulin resistance and hepatic steatosis in patients with severe lipodystrophy. J Clin Invest 2002;109:1345-1350.

5 Javor ED, Cochran EK, Musso C, Young JR, Depaoli AM, Gorden P: Long-term efficacy of leptin replacement in patients with generalized lipodystrophy. Diabetes 2005;54: 1994-2002.

6 Chong AY, Lupsa BC, Cochran EK, Gorden P: Efficacy of leptin therapy in the different forms of human lipodystrophy. Diabetologia 2010;53:27-35

7 Oral EA, Simha V, Ruiz E, Andewelt A, Premkumar A, Snell P, et al: Leptin-replacement therapy for lipodystrophy. N Engl J Med 2002;346:570-578.
8 Kleiner DE, Brunt EM, Van Natta M, Behling C, Contos MJ, Cummings OW, et al: Design and validation of a histological scoring system for nonalcoholic fatty liver disease. Hepatology 2005;41:1313-1321.

$>9$ Fiorenza CG, Chou SH, Mantzoros CS: Lipodystrophy: pathophysiology and advances in treatment. Nat Rev Endocrinol 2011;7:137-150.

10 Green ED, Maffei M, Braden VV, Proenca R, DeSilva U, Zhang Y, et al: The human obese (OB) gene: RNA expression pattern and mapping on the physical, cytogenetic, and genetic maps of chromosome 7. Genome Res 1995;5:5-12.

11 Klein S, Coppack SW, Mohamed-Ali V, Landt M: Adipose tissue leptin production and plasma leptin kinetics in humans. Diabetes 1996;45:984-987. 
12 Masuzaki H, Ogawa Y, Sagawa N, Hosoda K, Matsumoto T, Mise H, et al: Nonadipose tissue production of leptin: leptin as a novel placenta-derived hormone in humans. Nat Med 1997;3:1029-1033.

-13 Mizuno TM, Mobbs CV: Hypothalamic agouti-related protein messenger ribonucleic acid is inhibited by leptin and stimulated by fasting. Endocrinology 1999;140:814-817.

14 Caro JF, Sinha MK, Kolaczynski JW, Zhang PL, Considine RV: Leptin: the tale of an obesity gene. Diabetes 1996;45:1455-1462.

- 15 Licinio J, Mantzoros C, Negrao AB, Cizza G, Wong ML, Bongiorno PB, et al: Human leptin levels are pulsatile and inversely related to pituitary-adrenal function. Nat Med 1997;3:575-579.
6 Musso C, Cochran E, Javor E, Young J, Depaoli AM, Gorden P: The long-term effect of recombinant methionyl human leptin therapy on hyperandrogenism and menstrual function in female and pituitary function in male and female hypoleptinemic lipodystrophic patients. Metabolism 2005;54:255-263.

17 Eren E, Ozkan TB, Cakir ED, Saglam H, Tarim O: Acquired generalized lipodystrophy associated with autoimmune hepatitis and low serum C4 level. J Clin Res Pediatr Endocrinol 2010;2:39-42.

18 Savage DB, Semple RK, Clatworthy MR, Lyons PA, Morgan BP, Cochran EK, et al: Complement abnormalities in acquired lipodystrophy revisited. J Clin Endocrinol Metab 2009;94:10-16.
9 Farooqi IS, Matarese G, Lord GM, Keogh JM, Lawrence E, Agwu C, et al: Beneficial effects of leptin on obesity, $\mathrm{T}$ cell hyporesponsiveness, and neuroendocrine/metabolic dysfunction of human congenital leptin deficiency. J Clin Invest 2002;110:1093-1103.

20 Roth T, Nair S, Kumar A: Monogenic diabetes secondary to congenital lipodystrophy in a 14-year-old Yemeni girl. J Clin Res Pediatr Endocrinol 2010;2:176-179.

21 Tsai YS, Kim HJ, Takahashi N, Kim HS, Hagaman JR, Kim JK, et al: Hypertension and abnormal fat distribution but not insulin resistance in mice with P465L PPAR $\gamma$. J Clin Invest 2004;114:240-249. 